\title{
Redistribution and Subsidies for Higher Education
}

\author{
By CARMEN BEVIÁ and IÑIGO ITURBE-ORMAETXE * \\ Universitat Autònoma de Barcelona and CODE; Universidad de Alicante
}

January 2001

\begin{abstract}
The financing of higher education through public spending imposes a transfer of resources from taxpayers to the university students and their parents. We provide an explanation for this phenomenon. Those who attend higher education will earn more income in the future and will pay more taxes. People whose children do not attend higher education, however should agree to help pay the cost of such education, providing that the taxes are sufficiently high to ensure that there will be an adequate redistribution in favor of their own children at some time in the future.
\end{abstract}

Journal of Economic Literature classification numbers: D71, H21, H52.

Key words: Higher Education, Taxation, Redistribution.

\footnotetext{
${ }^{*}$ We would like to thank Luis C. Corchón for his many very useful comments. We are also greatful to Jordi Caballé, Ignacio Ortuño-Ortín, John E. Roemer and Guadalupe Valera for their helpful comments. We are very grateful to John E. Roemer for his hospitality under the program Economy, Justice and Society of the University of California at Davis. We thank the financial support from Instituto Valenciano de Investigaciones Económicas, DGICYT PB98-0870, PB94-1504 and PB97-0120 and Direcció General de Reserca under project SGR98-0062. Address for correspondence: Iñigo Iturbe-Ormaetxe, Departamento de Fundamentos del Análisis Económico, Universidad de Alicante, E-03071, Alicante, Spain. E-mail: iturbe@merlin.fae.ua.es
} 


\section{Introduction}

In most countries the cost of public higher education is financed mainly by the government out of general tax revenue. In Spain, for example, only about $20 \%$ of total cost was covered by the fees paid by students in 1996, while the remaining $80 \%$ was covered by public transfers (see Calero (1996)). It is also well known that most university students come from middle and upper income groups. Such empirical evidence has led some authors to conclude that public financing of higher education brings about a regressive effect on income distribution (see Hansen and Weisbrod (1969) and Nerlove (1972)). Leslie and Brinkman (1988), however, conclude that this regressive effect is offset once the progressive nature of the tax system is taken into account. The following question, however, remains to be answered: Since higher education is an activity that provides direct benefits by increasing future earning power, to subsidy higher education means to subsidy an activity that provides direct benefits to a privileged minority only. Why then, in a democratic society, should a majority of voters, who do not have access to higher education, agree to subsidize it for the wealthiest segment of the population? As this seems to be the case throughout western societies, many researchers have developed theories to explain this phenomenon. ${ }^{1}$

The explanation most frequently offered is that higher education creates "spillovers," or positive effects, for the rest of the economy. It would, therefore, be unfair to impose the entire cost of higher education on the students and their families. If this were done, families would tend to under-invest in higher education. Since those who receive higher education and generate the "spill-over" cannot force its indirect beneficiaries to pay for the benefits they receive, it can be accomplished through the government, via taxes.

An example on this vein is Johnson (1984), who suggests that education not only increases the productive skills of those who receive it, but can also indirectly benefit those who do not, via "complementarities" in the production process. Creedy and Francois (1990) develop a more complex model. They assume the existence of a positive effect of education on the growth rate of a country. This, in turn, increases the future income of the non-educated individuals, who, providing that the positive effect is great enough, will agree to pay higher taxes to continue financing higher education. However, as Creedy and Francois admit, there is no empirical evidence of the existence of such spill-overs, and far less of their degree. This explanation,

\footnotetext{
${ }^{1}$ We refer exclusively to "democratic" explanations. That is, those that are based on some type of collective decision, by majority vote. We could provide an alternative explanation for non-democratic societies: Political power is concentrated in the upper income group and they can extract the desired resources from the other groups through coercive taxation.
} 
therefore, may lose some of its appeal. ${ }^{2}$

Another explanation is that offered by Fernandez and Rogerson (1995). When people vote on the size of a subsidy for education, they are also voting, implicitly, on how many students should receive such a subsidy and attend university. A given amount of subsidy determines the proportion of people who can go to university: those above a certain income threshold. The level of this threshold is lower the greater is the size of the subsidy. These authors show that, in some cases, a majority of voters in the middle and high income brackets, can force the choice of a partial subsidy, and thus, exclude low-income groups from attending higher education while extracting resources from them, through the tax system.

Garratt and Marshall (1994) see the government as a provider of insurance. Only the most skilled individuals can attend college. If parents are uncertain about the ability of their children, they will be willing to insure themselves against the possibility of having able children and not being able to afford their education. Thus the families whose children do not attend college make payments as an insurance premium.

In this paper, we propose a different explanation for the public funding of higher education. As we have already mentioned, those who attend university will eventually earn, on average, more income than they would have earned if they had not gone to university. This, in turn, implies that the gross total income will increase, in the future, due to the existence of higher education and so will the tax base. Moreover, the greater the subsidy to higher education is, the greater the increase in future income will be, as there will be more young people attending university. Let us now suppose that transfers to low income households are positively related to the tax base. This means that today, families whose children have no access to higher education, can anticipate that their children will benefit in the future, since the transfers that are made to their benefit will be greater as tax collection increases with the rise in the number of students. Of course, the higher the marginal tax rate is, the greater the effect will be, as it will determine how much of the increase in income remains in private hands, (in other words, in the hands of those who went to university), and how much will be redistributed among the society. ${ }^{3}$ In other words, publicly subsidizing higher education eliminates deadweight loss caused by the tax system.

As parents care about the future income of their children, they will be willing to pay the extra taxes needed to finance a higher level of subsidy. As the level of the subsidy increases, more people will choose to go to the university and tax revenue from the former university students will grow.

\footnotetext{
${ }^{2}$ The available empirical evidence suggests that such spill-overs are high for primary and secondary education and low for higher education. See, for example, Psacharopoulos (1985).

${ }^{3}$ In order to avoid problems regarding the formation of expectations of future tax rates, we shall assume, throughout the paper, that all people believe that the future tax rate will be the same as the current rate.
} 
Our main argument here is that there is a positive relationship between the perceived degree of redistribution of taxes among a society, (measured by the marginal tax rate), and the level of the subsidy that is allocated to higher education. ${ }^{4}$ To sum up, the marginal tax rate determines what proportion of the increase in future income due to education is redistributed among the society and how much remains in private hands. This idea is not entirely new. It dates back, at least, to Nerlove (1972) who believed that part of the subsidy could be regained through higher taxes that should be levied on the upper-income levels, which are precisely those who have benefitted from the increased subsidies for higher education. Blomquist (1982) studies, in an optimal taxation framework, to what extent educational expenses should be deductible in order to maximize a Rawlsian social welfare function. Allen (1982) shows that, in some cases, the worst-off people are helped by a linear tax consisting of a wage subsidy and a uniform lump-sum tax, which redistributes from poor to rich. Barr (1993) states that if the subsidy to higher education were zero, future tax-payers would get a dividend, via the increase in the tax base, and thus, the government would eventually have to establish a subsidy to restore efficiency. Our argument is that this could be guaranteed, even in a situation in which the subsidy were chosen by majority vote, with the voters trying to maximize their own income levels. To make our point stronger, we consider the decisive voters to be those families who do not, or cannot, send their children to university. The main finding of our study is that those families would vote for a positive subsidy to higher education, and that such a subsidy would grow along with the level of the marginal tax rate. This contrasts with the results of Fernandez and Rogerson (1995), as in their case the decisive voters were always those who attend higher education.

In Section 2, we present the model and some preliminary results. Our model is a human capital model, as attendance to university adds to productivity. We assume that the society has to decide, by majority vote, the amount of the subsidy that is to be allocated to higher education. When a family votes, they consider the way in which a given level of subsidy determines the proportion of students that attend university. In Section 3, we study the relationship between the level of subsidy chosen by the society and the marginal tax rate. We find that, for reasonable values of the parameters, this relationship is always positive. That is to say, the greater the marginal tax rate is, the greater the level of the subsidy chosen by the society will be. Finally, in Section 4, we discuss some of the weaknesses of our model.

\footnotetext{
${ }^{4} \mathrm{~A}$ similar idea is explored in Bergstrom and Blomquist (1996), who propose a situation in which people could vote for higher subsidies for day care, as it would induce mothers to join the work force and, hence, pay income tax.
} 


\section{Preliminaries}

We present a model with two periods, labelled 0 and 1 . In period 0 there is a continuum of families that are composed of one parent and one offspring. Families are characterized by a pair $\left(y_{0}, \delta\right)$ where $y_{0} \in[0, \infty)$ is income earned by the parent in period 0 and $\delta \in[0, \infty)$ is the ability or "talent" of the child. We assume $y_{0}$ and $\delta$ are independently distributed. Suppose, for instance, that the particular value of $\delta$ depends on individual characteristics, such as intelligence, which we assume, a priori, are unrelated to income. We denote by $G\left(y_{0}\right)$ and $H(\delta)$ the cumulative density functions $(\mathrm{CDF})$ of $y_{0}$ and $\delta$ respectively $\left(g\left(y_{0}\right)\right.$ and $h(\delta)$ represent the density functions).

The total cost per student for the higher education system is $k+c$, where $k>0$ and $c>0$. The term $k$ represents the implicit cost, i.e., foregone earnings. For simplicity, we assume that $k$ is independent of the characteristics of the families. The term $c$ represents all explicit costs: tuition and fees, and room and board rates. There is a crucial difference in our model between $k$ and $c$. The implicit cost $k$ is not subsidized at all while $c$ can be subsidized by the government at a rate $s$, with $0 \leq s \leq 1$. In other words, families pay $k+(1-s) c$ if their offspring goes to university.

All decisions are made by the parents in period 0 . Specifically, they must make two decisions: First, they must decide, for a given $s$, whether or not they agree to pay $k+(1-s) c$. We assume that children are unable to borrow funds, and thus, cannot afford to pay their own fees, so that they can only attend university if their parents are willing to pay for it. Secondly, parents must decide collectively, by majority vote, the level of the subsidy that should be allocated to higher education.

In period 1, only the children live and their levels of income are determined both by the income levels of their parents and by their attendance or not at university. We assume that income in period $1, y_{1}$, for a child from a family with characteristics $\left(y_{0}, \delta\right)$ will comply with the following pattern: ${ }^{5}$

$$
y_{1}\left(y_{0}, \delta\right)= \begin{cases}y_{0} & \text { if the child does not attend university } \\ (1+\delta) y_{0} & \text { if the child attends university. }\end{cases}
$$

According to this pattern, the value of $\delta$ matters only when the child attends university. This amounts to say that $\delta$ is more a measure of ability to learn than a measure of talent. It captures differences in the increment to human capital from attending university.

\footnotetext{
${ }^{5}$ We assume that all students graduate. The return from education does not depend on the number of students. A more general treatment would allow for considering such a possibility, introducing wage adjustments to changes in the supply of graduate students.
} 


\subsection{Tax Structure}

Taxes are levied according to the following linear equations:

$$
t\left(y_{0}\right)=b_{0}+a y_{0} \text { where } b_{0} \leq 0 \text { and } 0<a<1 \text { in period } 0,
$$

and

$$
t\left(y_{1}\right)=b_{1}+a y_{1} \text { where } b_{1} \leq 0 \text { and } 0<a<1 \text { in period } 1 .
$$

The marginal tax rate $a$ is the same during both periods and is fixed by the government. The lump-sum transfers $-b_{0}$ and $-b_{1}$ will vary to satisfy the budget constraints of the government (see Subsection 2.3 below). These transfers can be seen as the guaranteed minimum income for every individual. They are assumed to be non-negative to ensure that the tax function is progressive.

\subsection{Parents' First Decision}

Here, we take the subsidy level $s$ as given. We assume that the parents' utility is simply a weighted sum of after-tax income during period 0 and after-tax income of their offspring during period 1.If the child attends university, the utility that the parent of a family $\left(y_{0}, \delta\right)$ enjoys is:

$$
v\left(s ; y_{0}, \delta\right)=(1-a) y_{0}-b_{0}-k-(1-s) c+\lambda\left\{(1-a)(1+\delta) y_{0}-b_{1}\right\} .
$$

From after-tax income during period 0 we subtract $k+(1-s) c$, which represents the share of the cost of higher education that the family has to contribute.

The parameter $\lambda>0$ expresses the rate at which parents discount their children's income. The greater is $\lambda$, the more weight they give to the future income of the children. This parameter can be seen as the degree of parents' altruism. They will agree to pay $\lambda$ dollars of their after-tax income during period 0 if, by doing so, the after-tax income of their offspring would increase by 1 dollar during period 1 . If $\lambda<1$ parents put more weight on their own income than on the future incomes of their children. If the child does not attend university, the utility is:

$$
u\left(s ; y_{0}, \delta\right)=(1-a) y_{0}-b_{0}+\lambda\left\{(1-a) y_{0}-b_{1}\right\}
$$

Parents will agree to pay the cost of university education provided $v\left(s ; y_{0}, \delta\right) \geq$ $u\left(s ; y_{0}, \delta\right)$. This will be the case when the discounted post-tax increase in their child's income, thanks to higher education, is equal to or greater than the total cost borne by the family. That is:

$$
\lambda(1-a) \delta y_{0} \geq(1-s) c+k .
$$

Note that, even when $s=1$, not all families are willing to send their children to university, as would be the case if $k=0$. Certainly, if $s=1$ and $k=0$, total cost of education would be zero. 
Let $w(s, a)=\frac{(1-s) c+k}{\lambda(1-a)}$. This is a cut-off value for $\delta y_{0}$. The value $(1-a) w(s, a)$ represents the net cost of education, in future value. Therefore, the families who want to have their children at university are those with characteristics $\left(y_{0}, \delta\right)$ satisfying:

$$
\delta y_{0} \geq w(s, a) .
$$

As $\lambda>0$ and $a<1$ the term $w(s, a)$ is well-defined. Note that $w(s, a)$ increases with $c, k$ and $a$, and decreases with $s$ and $\lambda$. Furthermore, for given $c, k, \lambda$ and $a$, $w(s, a) \in\left[\frac{k}{\lambda(1-a)}, \frac{c+k}{\lambda(1-a)}\right]$.

We define $\widehat{s}\left(y_{0}, \delta\right)$ as the minimum value of the subsidy $s$ that a family of characteristics $\left(y_{0}, \delta\right)$ requires to be willing to send their child to university. From the above inequality we have:

$$
\widehat{s}\left(y_{0}, \delta\right)=1-\left[\frac{\lambda(1-a) \delta y_{0}-k}{c}\right] .
$$

According to the value of $\widehat{s}\left(y_{0}, \delta\right)$ the population can be partitioned into three groups:

(i) Those with $\widehat{s}\left(y_{0}, \delta\right)>1$, or $\delta y_{0}<w(1, a)$.

(ii) Those with $\widehat{s}\left(y_{0}, \delta\right)<0$, or $\delta y_{0}>w(0, a)$.

(iii) Those with $0 \leq \widehat{s}\left(y_{0}, \delta\right) \leq 1$, or $w(1, a) \leq \delta y_{0} \leq w(0, a)$.

Group (i) contains all the families that would require a greater subsidy than the maximum $(s=1)$ for their child to have access to higher education. In other words, it contains all the families who will never enjoy higher education. Group (ii) reflects the families that, contrary to (i), will be willing to send their child to university even when $s=0$. Finally, group (iii) is made up of all those families for whom their decision is not independent of $s$, as it is for those in Groups (i) and (ii). They will only send their child to university if $s \geq \widehat{s}\left(y_{0}, \delta\right)$.

We compute the proportion of families who wish to send their offspring to university. We write this proportion as a function of $s$ and $a$ :

$$
p(s, a)=\operatorname{Pr}[z \geq w(s, a)]=1-F(w(s, a))=\int_{w(s, a)}^{+\infty} d F(z),
$$

in which $z=\delta y_{0}$ and $F(z)$ is the $\mathrm{CDF}$ of $z\left(f(z)\right.$ its density function). ${ }^{6}$ It is important to remark that $p(s, a)$ increases with $s$ and decreases with $a$. This point makes the basic trade-off of the paper.

\footnotetext{
${ }^{6}$ If, for instance, both $\delta$ and $y_{0}$ follow a Log-normal distribution this is also the case for $z$. In particular if $\delta \backsim L N\left(\mu_{\delta}, \sigma_{\delta}^{2}\right)$ and $y_{0} \backsim L N\left(\mu_{y}, \sigma_{y}^{2}\right)$ then $z \backsim L N\left(\mu_{z}, \sigma_{z}^{2}\right)$ where $\mu_{z}=\mu_{\delta}+\mu_{y}$ and $\sigma_{z}^{2}=\sigma_{\delta}^{2}+\sigma_{y}^{2}$.
} 


\subsection{The Government's Budget Constraints}

We assume that the government cannot transfer funds from one period to another. The reason for this is that our two period model can be seen as a simplification for the steady state in a multi-period model. We also assume that, apart from redistribution, the only other expenditure is the subsidy to be allocated to higher education. In period 0 the budget constraint is:

$$
\int_{0}^{\infty}\left(a y_{0}+b_{0}\right) d G\left(y_{0}\right)=s c p(s, a) .
$$

The term on the right represents total subsidies. From this equation we can obtain the value of $b_{0}$ that balances the constraint:

$$
b_{0}(s, a)=\operatorname{scp}(s, a)-a \bar{y}_{0},
$$

where $\bar{y}_{0}$ is the mean income in period 0 . As $b_{0}(s, a)$ increases in $s$, to ensure that $b_{0}(s, a) \leq 0$ for all $s$ we must assume that $a \geq \frac{c}{\bar{y}_{0}}$. In other words, we need a lower boundary for $a$ that is higher than zero. The fact that $b_{0}(s, a)$ is increasing in $s$ means that the lump-sum transfer to all parents $\left(-b_{0}(s, a)\right)$, decreases with $s$. This implies a reduction in the guaranteed minimum income.

In Period 1, the government collects taxes for redistribution purposes only. The value of $b_{1}$ that balances the constraint is:

$$
b_{1}(s, a)=-a \bar{y}_{1}(s, a)
$$

where $\bar{y}_{1}(s, a)$ is mean income in period 1 and is equal to:

$$
\overline{y_{1}}(s, a)=\overline{y_{0}}+\int_{w(s, a)}^{+\infty} z d F(z) .
$$

As $\overline{y_{1}}(s, a)$ increases in $s, b_{1}(s, a)$ decreases in $s$. That is, as $s$ increases with $a$ fixed, the guaranteed minimum income in period $1\left(-b_{1}(s, a)\right)$ will be larger.

\subsection{Parents' Political Decision}

Here we study which level of subsidy will be chosen collectively. To do so, we assume that every family, when confronted with a choice between two different levels of subsidy, will vote for the one that maximizes their indirect utility function. We require that the level of subsidy chosen by the society must be a Condorcet winner. We call the level $s^{*}$ a Condorcet winner if, for all $s \neq s^{*}, U\left(s^{*} ; y_{0}, \delta\right) \geq U\left(s ; y_{0}, \delta\right)$ for at least half of the population.

Let $U\left(s ; y_{0}, \delta\right)$ be the indirect utility function of a family with the characteristics $\left(y_{0}, \delta\right)$. This function will be different for each of the three types of families that we had according to the particular values of $\widehat{s}\left(y_{0}, \delta\right)$. First, families with $\widehat{s}\left(y_{0}, \delta\right)>1$ 
will have $U\left(s ; y_{0}, \delta\right)=u\left(s ; y_{0}, \delta\right)$ for all $s \in[0,1]$. Second, families with $\widehat{s}\left(y_{0}, \delta\right)<0$, $U\left(s ; y_{0}, \delta\right)=v\left(s ; y_{0}, \delta\right)$ for all $s \in[0,1]$. Third, for those families with $0 \leq \widehat{s}\left(y_{0}, \delta\right) \leq 1$ :

$$
U\left(s ; y_{0}, \delta\right)= \begin{cases}u\left(s ; y_{0}, \delta\right) & \text { for } \quad 0 \leq s<\widehat{s}\left(y_{0}, \delta\right) \\ v\left(s ; y_{0}, \delta\right) & \text { for } \quad \widehat{s}\left(y_{0}, \delta\right) \leq s \leq 1\end{cases}
$$

Empirical evidence suggests that one of these groups, namely the first one, constitutes a majority. Such is the case when, even in the most favorable case, at least half of the families will not be able to send their children to university. This, in turn, means that the proportion $p(s, a)$ is bounded above by $\frac{1}{2}$. In Spain, for example, with a subsidy of about 0.8 , in 1995, $77.2 \%$ of the young people between 15 and 24 years were not at university (OECD average $82 \%$ ). The next assumption introduces some restrictions on the primitives of the model that lead us to a result that fits that empirical evidence.

Assumption 1 (i) $f(z)$ is unimodal. (ii) $\operatorname{Mode}(z) \leq \operatorname{Median}(z)<w(s, a)$.

The fact that $\operatorname{Mode}(z) \leq \operatorname{Median}(z)$, under (i), means that the Median $(z)$ is not in the increasing part of $f(z)$. The condition that Median $(z)<w(s, a)$ guarantees that $p(s, a)<\frac{1}{2}$. To illustrate this assumption, suppose $z$ is Log-normal. Then (i) holds true. It is also true that $\operatorname{Mode}(z) \leq \operatorname{Median}(z)$. Moreover, Median $(z)=$ $\exp \left(\mu_{z}\right)$, where $\mu_{z}$ is the mean in logarithms of $z$. As $w(s, a)$ takes values in the interval $\left[\frac{k}{\lambda(1-a)}, \frac{c+k}{\lambda(1-a)}\right]$, the second part of (ii) requires that $a \geq 1-\frac{k}{\lambda} \exp \left(-\mu_{z}\right)$. Recall that the progressiveness of the tax system required also $a \geq \frac{c}{\bar{y}_{0}}$. Putting together these two restrictions, we have that the marginal tax rate must satisfy $a \geq a_{\min }=\max \left\{\frac{c}{\bar{y}_{0}}, 1-\frac{k}{\lambda} \exp \left(-\mu_{z}\right)\right\}$.

If the first group of families contains at least half of the population, we can prove that a Condorcet winner always exists. To see this, note that all members in that group have utility functions as follows:

$$
U\left(s ; y_{0}, \delta\right)=u\left(s ; y_{0}, \delta\right)=(1+\lambda)(1-a) y_{0}-b_{0}(s, a)-\lambda b_{1}(s, a) .
$$

Only the first term of the utility function depends on the characteristics of the family. This is due to the additive form of the utility function. Then, all the utility functions of the individuals in that group will reach a maximum at the same value of $s$, that we call $s_{1}$. This will be the Condorcet winner. This value $s_{1}$ is the one that maximizes tax revenue, net of subsidies received, from the group that might send their children to university.

Once we know that a Condorcet winner exists, the next step is to check whether it is strictly positive or not. As the function $u\left(s ; y_{0}, \delta\right)$ is continuous in the whole interval $[0,1]$, a sufficient condition is that the first derivative of $u\left(s ; y_{0}, \delta\right)$ at the point $s=0$, is strictly positive. This will be the case if and only if:

$$
a \lambda \frac{\partial \overline{y_{1}}(0, a)}{\partial s}>c p(0, a) \text {. }
$$


This condition says that the discounted increase in the future tax collection due to a marginal increase in the subsidy must be higher than the increase in the total cost of higher education. If the function $u\left(s ; y_{0}, \delta\right)$ is strictly concave, this condition is also necessary. For given values of $\lambda$ and $c$, the condition will hold whenever the marginal tax rate $a$ lies above some threshold value. In the next section we compute, for reasonable values of the parameters, this threshold value of $a$, that we call $a_{l}$ (see Table 2 below).

Having a Condorcet winner which is strictly positive is not enough for our purposes, since we want to perform some exercises in comparative statics. Strict concavity of $u\left(s ; y_{0}, \delta\right)$ would help. The next result gives a condition under which $u\left(s ; y_{0}, \delta\right)$ is strictly concave on $s$ (see the Appendix for a proof).

Proposition 1 Suppose that Assumption 1 holds. Moreover, suppose the following condition holds:

$$
\lim _{z \rightarrow+\infty}\left(-\frac{f^{\prime}(z)}{f(z)}\right)=0 .
$$

Then there is some value of the marginal tax rate $\widehat{a}$, where $0<\widehat{a} \leq 1$, such that if $a \leq \widehat{a}$ both $v\left(s ; y_{0}, \delta\right)$ and $u\left(s ; y_{0}, \delta\right)$ are strictly concave functions on $s$.

Consider again the Log-normal case. The ratio $-\frac{f^{\prime}(z)}{f(z)}$ is equal to $\frac{\ln (z)-\mu_{z}+\sigma_{z}^{2}}{z \sigma_{z}^{2}}$, and the condition holds. To illustrate further, if $z$ follows a Pareto distribution with shape parameter $d>0$, the ratio $-\frac{f^{\prime}(z)}{f(z)}$ is $\frac{d+1}{z}$ and the condition also holds. Finally, we want to stress that $a \leq \widehat{a}$ is a sufficient, but not necessary, condition for strict concavity.

To illustrate the proposition and the restrictions on the parameters, we take US data from 1989 , when the median income was $\$ 28,906$ while mean income was $\$ 36,250$. Accordingly, we specify $y_{0} \backsim L N(3.36,0.4624)$. With respect to $\delta$ we propose a median value of 0.4 and a mean value of 0.5 . This implies that college graduates obtain, on average, a wage premium of $50 \%$ over those who do not graduate. Then, $\delta \backsim L N(-0.92,0.4463)$ and $z \backsim L N(2.44,0.9087)$. The National Center for Educational Statistics collects data on the costs of higher education. In 1989-90, average undergraduate tuition and fees were $\$ 1,356$ in public institutions, while average room and board rates were $\$ 1,513$ and $\$ 1,635$, respectively. ${ }^{7}$ Then we fix $c=\$ 4,504$. Finally we set $k=\$ 8,000$. With these data we have that $\frac{c}{\overline{y_{0}}}=0.124$. We also compute the values of $a_{\min }$ and $\widehat{a}$ for different values of $\lambda$. We collect them in Table 1 .

\footnotetext{
${ }^{7}$ See http://nces.ed.gov/pubs/digest97/d97t312.html
} 
Table 1

Extreme values of $a$

\begin{tabular}{l|cc}
\hline \hline \multicolumn{1}{c|}{$\lambda$} & $a_{\min }$ & $\widehat{a}$ \\
\hline \hline 0.25 & 0.124 & 0.44 \\
0.5 & 0.124 & 0.52 \\
0.75 & 0.124 & 0.57 \\
1 & 0.303 & 0.61 \\
\hline \hline
\end{tabular}

In what follows, we will assume that the conditions of Assumption 1 and Proposition 1 hold, and that $a \leq \widehat{a}$.

\section{Comparative Statics}

In this section, we assume that $0<s_{1}<1$. Therefore $s_{1}$ must satisfy the first order condition:

$$
\frac{\partial u\left(s_{1} ; y_{0}, \delta\right)}{\partial s}=-\frac{\partial b_{0}\left(s_{1}, a\right)}{\partial s}-\lambda \frac{\partial b_{1}\left(s_{1}, a\right)}{\partial s}=0 .
$$

This condition says that $s_{1}$ must be such that, at the margin, the negative effect of the subsidy on the guaranteed minimum income for the parents counterbalances the positive effect of the subsidy on average income of the children. Another way of writing this condition is:

$$
-c p\left(s_{1}, a\right)-c s_{1} \frac{\partial p\left(s_{1}, a\right)}{\partial s}+a \lambda \frac{\partial \overline{y_{1}}\left(s_{1}, a\right)}{\partial s}=0
$$

In our model, the marginal tax rate can be seen as a measure of future redistribution. Higher education increases future income, but from every additional dollar a given individual obtains, she must pay $a$ in taxes. We want to see if a higher value of $a$ yields a higher value of the subsidy. To study this effect of $a$, we apply the Implicit Function Theorem to obtain:

$$
\frac{\partial s_{1}}{\partial a}=-\frac{\frac{\partial^{2} u\left(s_{1} ; y_{0}, \delta\right)}{\partial s \partial a}}{\frac{\partial^{2} u\left(s_{1} ; y_{0}, \delta\right)}{\partial s^{2}}} .
$$

As we are assuming that $u\left(s ; y_{0}, \delta\right)$ is strictly concave, the sign of $\frac{\partial s_{1}}{\partial a}$ is the sign of $\frac{\partial^{2} u\left(s_{1} ; y_{0}, \delta\right)}{\partial s \partial a}$. This derivative can be written as:

$$
-c \frac{\partial p\left(s_{1}, a\right)}{\partial a}-c s_{1} \frac{\partial^{2} p\left(s_{1}, a\right)}{\partial s \partial a}+\lambda \frac{\partial \overline{y_{1}}\left(s_{1}, a\right)}{\partial s}+a \lambda \frac{\partial^{2} \overline{y_{1}}\left(s_{1}, a\right)}{\partial s \partial a},
$$

or, in terms of the function $p(s, a)$ only, as:

$$
-c \frac{\partial p\left(s_{1}, a\right)}{\partial a}+\lambda\left[w\left(s_{1}, a\right)+a \frac{\partial w\left(s_{1}, a\right)}{\partial a}\right] \frac{\partial p\left(s_{1}, a\right)}{\partial s}+\left[a \lambda w\left(s_{1}, a\right)-c s_{1}\right] \frac{\partial^{2} p\left(s_{1}, a\right)}{\partial s \partial a} .
$$


The first and second terms are positive. The first term reflects the fact that an increase in $a$ will reduce the proportion of students because families forecast that the post-tax return from higher education will shrink. Its effect is positive, since $s$ can be higher without increasing the total budget of higher education. The second term reflects the discounted positive impact on tax collection in the future. The third term, which is a second order effect, may be either positive or negative. In general, $\left[a \lambda w\left(s_{1}, a\right)-c s_{1}\right]$ will be negative when $a$ is low and positive when $a$ is large. The sign of the cross derivative of $p\left(s_{1}, a\right)$ is ambiguous. However we should expect it to be negative. This means that as $a$ grows, $p(s, a)$ becomes less sensitive to variations in $s$. In particular, this is the case when $z$ follows a Lognormal distribution and $w\left(s_{1}, a\right)>\operatorname{Median}(z)$. Then $\frac{\partial^{2} p\left(s_{1}, a\right)}{\partial s \partial a}=\frac{c}{\lambda(1-a)^{2}}\left[f\left(w\left(s_{1}, a\right)\right)+f^{\prime}\left(w\left(s_{1}, a\right)\right) w\left(s_{1}, a\right)\right]$ and the sign of $f\left(w\left(s_{1}, a\right)\right)+f^{\prime}\left(w\left(s_{1}, a\right)\right) w\left(s_{1}, a\right)$ is the sign of $\mu_{z}-\ln \left(w\left(s_{1}, a\right)\right)$.

Summing up, we have two first order effects that are positive, and one second order effect whose sign is ambiguous. If the first order effects dominate, the effect of $a$ on $s_{1}$ will be positive. To verify this, we calculate the value of $s_{1}$ for different values of the parameters. In every case, we have found a positive relation between $s_{1}$ and $a$. In fact, we have not found a single case in which $s_{1}$ decreases with $a$ (see Table 2). For each value of $\lambda$ in the table, we also compute two reference values of $a, a_{l}$ and $a_{h}$. The first one, $a_{l}$, is the maximum value of $a$ for which $s_{1}$ is zero. The second one, $a_{h}$, is the minimum value of $a$ for which $s_{1}$ is one.

Table 2

Optimal value of $s_{1}$ for different combinations of $a$ and $\lambda^{8}$

\begin{tabular}{|c|c|c|c|c|}
\hline & $\lambda=0.25$ & $\lambda=0.5$ & $\lambda=0.75$ & $\lambda=1$ \\
\hline$a=0.124$ & $0(0.038)$ & $0(0.157)$ & $0(0.288)$ & \\
\hline$a=0.15$ & $0(0.036)$ & $0(0.15)$ & $0(0.277)$ & \\
\hline$a=0.35$ & $0.356(0.0259)$ & $0.07(0.096)$ & $0(0.188)$ & $0(0.284)$ \\
\hline$a=0.45$ & $0.82(0.029)$ & $0.622(0.108)$ & $0.43(0.188)$ & $0.24(0.256)$ \\
\hline$a=0.6$ & $1(0.0163)$ & $1(0.085)$ & $1(0.176)$ & $1(0.27)$ \\
\hline$a_{l}$ & $0.278(0.024)$ & $0.338(0.095)$ & $0.381(0.174)$ & $0.415(0.2467)$ \\
\hline$a_{h}$ & $0.49(0.03)$ & $0.525(0.118)$ & $0.551(0.211)$ & $0.572(0.295)$ \\
\hline
\end{tabular}

Assuming a positive effect of $a$ on $s_{1}$, that is, assuming that $s_{1}(a)$ is a nondecreasing function on $a$, it is interesting to study the total effect that the marginal tax rate has on mean income in Period 1 , which we can write as $\overline{y_{1}}\left(s_{1}(a), a\right)$. For the sake of simplicity, and building on the figures presented in Table 2, we assume that $a_{l}$ and $a_{h}$ are such that:

\footnotetext{
${ }^{8}$ The values of the parameters are $c=\$ 4,504, k=\$ 8,000, z \sim L N(2.44,0.95)$. The numbers in brackets are the proportions of students for the corresponding values of the parameters.
} 
(i) $a_{\min } \leq a_{l}<a_{h} \leq 1$.

(ii) For all $a \leq a_{l}, s_{1}(a)=0$.

(iii) For all $a \geq a_{h}, s_{1}(a)=1$.

(iv) For all $a$ such that $a_{l}<a<a_{h}, 0<s_{1}(a)<1$.

Now we recall how $\overline{y_{1}}(s, a)$ was defined in Section 2. It is the integral of some function that does not depend on either $s$ or $a$. Only $w(s, a)$ depends on such parameters. But then, all combinations $(s, a)$ for which $w(s, a)$ is constant, give rise to the same value of $\overline{y_{1}}(s, a)$.

In the first panel of Figure 1, we draw some level curves of $\overline{y_{1}}(s, a)$ in the space $(a, s)$. They are straight lines with slope $\frac{\lambda}{c} w(s, a)=\frac{(1-s) c+k}{c(1-a)}$. In what follows, we shall refer to these lines as constant-income lines. Note that the greater $a$ is, the greater the slope of the constant-income lines will be. This means that the increase required in $s$ to offset an increase in $a$, while leaving $\overline{y_{1}}(s, a)$ unchanged, increases with $a$. The bold line in the figure represents $s_{1}(a)$.

It is interesting to note that, both to the left of $a_{l}$ and to the right of $a_{h}$, the function $\overline{y_{1}}\left(s_{1}(a), a\right)$ decreases in $a$. The reason for this is that, in these intervals, $s_{1}(a)$ is constant (either at 0 or at 1 ), and thus, any increase in $a$ lowers mean income in Period 1.

Let us define $a^{+}$as the value of $a$ at which $\overline{y_{1}}\left(s_{1}(a), a\right)$ attains a maximum in the interval $\left[a_{l}, a_{h}\right]$ (see Figure 1). This is always well-defined. We want to check whether $a^{+}$is a global maximum of $\overline{y_{1}}\left(s_{1}(a), a\right)$ on the interval $\left[a_{\min }, 1\right]$. This will not be the case when $a^{+}=a_{l}$. In this case the maximum of $\overline{y_{1}}\left(s_{1}(a), a\right)$ will be attained at $a=a_{\min }$ with a subsidy of zero. Let us now suppose $a^{+}>a_{l}$ as it happens in Figure 1 . The following proposition gives a condition under which $\overline{y_{1}}\left(s_{1}(a), a\right)$ attains a maximum at $a^{+}$.

Proposition 2 Let $a_{l}, a_{h}$ and $a^{+}$be as defined above. Then $\overline{y_{1}}\left(s_{1}(a), a\right)$ will attain a maximum at $a^{+}$if and only if $s_{1}\left(a^{+}\right)>\left(1+\frac{k}{c}\right)\left(\frac{a^{+}-a_{\min }}{1-a_{\min }}\right)$.

Proof. All we need to prove is that, under the above condition, $\overline{y_{1}}\left(s_{1}(a), a\right)$ takes a higher value at $a^{+}$than at $a_{\text {min }}$. As we already know that $s_{1}\left(a_{\min }\right)=0$, what we need to prove is that the constant-income line that passes through $\left(a^{+}, s_{1}\left(a^{+}\right)\right)$represents a higher value of $\overline{y_{1}}\left(s_{1}(a), a\right)$ than the constant-income line that passes through $\left(a_{\min }, 0\right)$. Or alternatively, that $\widehat{s}>0$ where $\widehat{s}$ is defined such that $\overline{y_{1}}\left(\widehat{s}, a_{\min }\right)=$ $\overline{y_{1}}\left(s_{1}\left(a^{+}\right), a^{+}\right)$. At the point $\left(s_{1}\left(a^{+}\right), a^{+}\right)$the slope of the constant-income line will be $\beta^{+}=\frac{\left(1-s_{1}\left(a^{+}\right)\right) c+k}{c\left(1-a^{+}\right)}$. Therefore, $\widehat{s}=s_{1}\left(a^{+}\right)+\beta^{+}\left(a_{\min }-a^{+}\right)$. The value $\widehat{s}$ will be greater than zero if, and only if, $s_{1}\left(a^{+}\right)>\left(1+\frac{k}{c}\right)\left(\frac{a^{+}-a_{\min }}{1-a_{\min }}\right)$. Note that, in particular, this is what happens in Figure 1. 
For fixed values of $a_{\min }$ and $a^{+}$, the condition in the proposition will hold whenever $\frac{k}{c}$ is sufficiently low, in which case the constant-income lines become sufficiently flatter. Recall that $k$ represents the unsubsidized costs. For a high enough ratio between implicit and explicit costs, it may be that the value of $a$ that maximizes $\overline{y_{1}}\left(s_{1}(a), a\right)$ is $a_{\mathrm{min}}$, at which point we already know that there will be zero subsidy. An increase of the tax rate from $a_{\text {min }}$ to $a^{+}$, although it raises $s$, does not reduce education costs by much. The increase in $s$ reduces only the explicit (subsidized) costs which are, in this case, a small fraction of the total costs. In particular this was the case in the examples of Table 2.

In the second panel of Figure 1 we also represent the values of $\overline{y_{1}}\left(s_{1}(a), a\right)$ for any value of $a$, once we take the voting behavior of the population into account. Note that it is quite similar to a Laffer curve. The difference is that, in our model, the efficiency costs arise, not because of the existence of distortions in the labor supply, but because taxes reduce the demand for education. Moving to the right of $a^{+}$, keeps on increasing $s$, but this increase is not enough to offset the negative impact of the increase in the tax rate.

\section{Figure 1}

\section{Final Comments and Criticisms}

In our model there are only two ways for parents of transferring resources to their children. One is to pay for the education of their own children. The other is to invest in other families' children and, in that way, influence the size of the guaranteed minimum income the children will obtain in the future. We prove that this second type of investment will be carried out, provided that the future marginal tax rate is high enough. One possible difficulty with this is the following. Suppose parents could transfer resources to their children via cash. Would the results still maintain? The answer is positive, but this is due to the form of the utility functions. As long as $a>0$ and $\lambda<1$, the optimal bequest is always zero. To address properly this problem we would need a more sophisticated model, at the risk of facing a problem of non existence of a voting equilibrium.

The subsidies we have studied in this paper are very simplistic. They are conveniently independent of income. In general, however, subsidies are income-dependent. Students from low-income families receive grants. Nevertheless, once income has reached a certain level, the subsidy is constant: people in the middle-income group who are not eligible for grants, pay the same fees as those in higher, and even much higher income levels. In any case, the introduction of subsidies dependent on income would complicate the problem, since, in such a case, voters would have to choose policies from a multi-dimensional space, giving rise to the usual problem of non-existence of equilibrium. 
In this paper we have made allowance for people to vote on the amount of the subsidy, while the level of the marginal tax rate remains fixed. If people are also allowed to vote on the level of the marginal tax rate, we come up against a similar problem to the one mentioned above.

Another related weakness in our model is that voters believe that the marginal tax rate will remain unchanged in the future. Note that in our model everything depends on the future tax rate rather than on the current one. To be more specific, what is the expected future tax rate is what matters most. To deal adequately with this problem, a detailed description of how expectations on the future tax rate are formed would be required. In our model, we choose the simplest possible way of doing this. We assume that everybody believes that the marginal tax rate will be exactly what the current one is.

Finally, the main drawback of the model is its static nature. In a dynamic framework, voters should take into account that the positive effect of $s$ on future income may also, in the next period, increase the proportion of students, making the given subsidy more costly. The rise in the cost of education will reduce the guaranteed minimum income. These two effects have opposite signs and the final result might be ambiguous. 


\section{APPENDIX}

\section{Proof of Proposition 1}

We want to study conditions under which:

$$
u^{\prime \prime}(s)=-c\left(2 \frac{\partial p(s, a)}{\partial s}+s \frac{\partial^{2} p(s, a)}{\partial s^{2}}\right)+a \lambda \frac{\partial^{2} \overline{y_{1}}(s, a)}{\partial s^{2}}<0 .
$$

After substituting the expressions of $\frac{\partial p(s, a)}{\partial s}, \frac{\partial^{2} p(s, a)}{\partial s^{2}}$ and $\frac{\partial^{2} \overline{y_{1}}(s, a)}{\partial s^{2}}$ and rearranging terms, this is the same as:

$$
\lambda(2-a) f(w(s, a))>(s c-a \lambda w(s, a)) f^{\prime}(w(s, a)),
$$

or

$$
\lambda(2-a) f(w(s, a))>\frac{s c-a(c+k)}{(1-a)} f^{\prime}(w(s, a)) .
$$

By Assumption 1, $f^{\prime}(w(s, a))<0$. If $s c-a(c+k) \geq 0$ or $a \leq \frac{c}{c+k} s$ we are done. Then, suppose that $s c-a(c+k)<0$. We have to prove that:

$$
-\frac{f^{\prime}(w(s, a))}{f(w(s, a))}<\frac{\lambda(2-a)(1-a)}{a(c+k)-s c} .
$$

Call $B(s, a)=\frac{\lambda(2-a)(1-a)}{a(c+k)-s c}$. We know that for all $s$ and for all $a>\frac{c}{c+k} s, B(s, a) \geq 0$, $\frac{\partial B(s, a)}{\partial a}<0, \frac{\partial^{2} B(s, a)}{\partial a^{2}}>0$ and $\frac{\partial B(s, a)}{\partial s}>0$. Moreover $\lim _{a \rightarrow \frac{c}{c+k} s} B(s, a)=+\infty$ and $\lim _{a \rightarrow 1} B(s, a)=0$ for all $s$. Now call $M(s, a)=-\frac{f^{\prime}(w(s, a))}{f(w(s, a))}$. We have that $M(s, a) \geq 0$ for all $(s, a)$ and $0<M\left(s, \frac{c}{c+k} s\right)<+\infty$ for all $s$. By the condition in the proposition, we know that $\lim _{a \rightarrow 1} M(s, a)=0$. Now we fix $s$. As both $B(s, a)$ and $M(s, a)$ are continuous on $a$ there are two possible cases: (i) $B(s, a)$ and $M(s, a)$ cross at some value (or values) of $a$; (ii) $M(s, a)$ is below $B(s, a)$ for all $a$. If they cross, we call $\widehat{a}(s)$ the minimum value of $a$ at which those functions cross. In the second case we set $\widehat{a}(s)=1$. Finally we take $\widehat{a}=\min _{s}\{\widehat{a}(s)\}$. By construction, it must be that for all $a<\widehat{a}, u^{\prime \prime}(s)=v^{\prime \prime}(s)<0$. In general, we cannot say anything about the relationship between $\widehat{a}(s)$ and $s$. However, if we consider the Lognormal case, then $\widehat{a}(s)$ is an increasing function on $s$ and thus, $\widehat{a}=\widehat{a}(0)$. 


\section{References}

[1] Allen, F., "Optimal Linear Income Taxation with General Equilibrium Effects on Wages," Journal of Public Economics 17 (1982), 135-143.

[2] Barr, N., "Alternative Funding Resources for Higher Education," Economic Journal 103 (1993), 718-728.

[3] Bergstrom, T. and S. Blomquist, "The Political Economy of Subsidized Day Care," European Journal of Political Economy 12 (1996), 443-457.

[4] Blomquist, S., "Should Educational Expenses be Deductible?," Journal of Public Economics 18 (1982), 65-83.

[5] Calero, J., Financiación de la educación superior en España: sus implicaciones en el terreno de la equidad, (Bilbao: Fundación BBV, 1996).

[6] Creedy, J. and P. Francois, "Financing Higher Education and Majority Voting," Journal of Public Economics 43 (1990), 181-200.

[7] Fernandez, R. and R. Rogerson, "On the Political Economy of Education Subsidies," Review of Economic Studies 62 (1995), 249-262.

[8] Garratt, R. and J. Marshall, "Public Finance of Private Goods: The Case of College Education," Journal of Political Economy 102 (1994), 566-582.

[9] Johnson, G. E., "Subsidies for Higher Education," Journal of Labor Economics 2, 3 (1984), 303-318.

[10] Hansen, W. L. and B. A. Weisbrod, "The Distribution of the Costs and Direct Benefits of Public Higher Education," Journal of Human Resources 4, 2 (1969), 176-191.

[11] Leslie, L. L. and P. T. Brinkman, "Equitability in Traditional Financial Support Systems," in L. Leslie and P. Brinkman, eds., The Economic Value of Higher Education (London: MacMillan, 1988), cap. 6, pp. 107-121.

[12] Nerlove, M., "On Tuition and the Costs of Higher Education: Prolegomena to a Conceptual Framework," Journal of Political Economy 80, 3, Part II (1972), s178-s218.

[13] Psacharopoulos, G., "Returns to Education: A Further International Update and Implications," Journal of Human Resources 20 (1985), 583-597. 
Level of the subsidy $s$

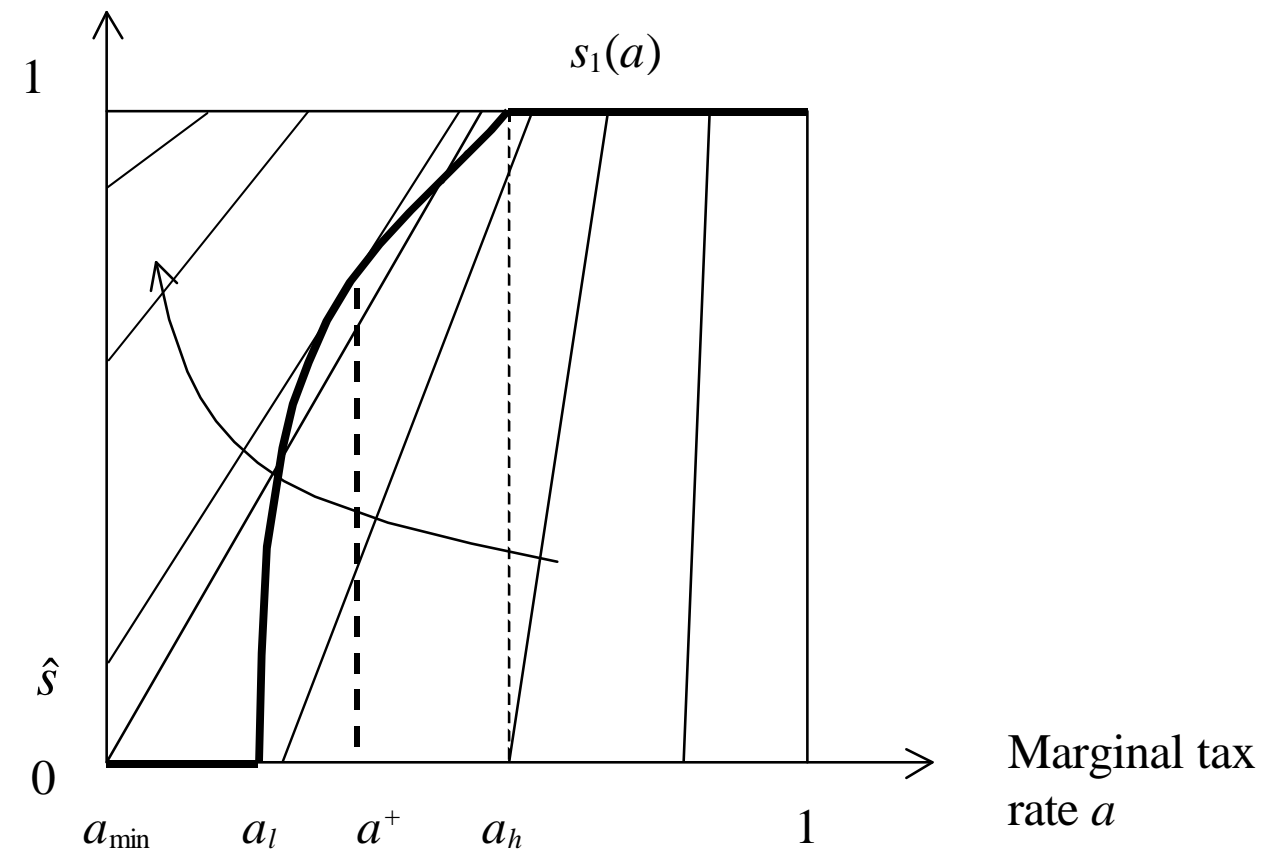

Mean

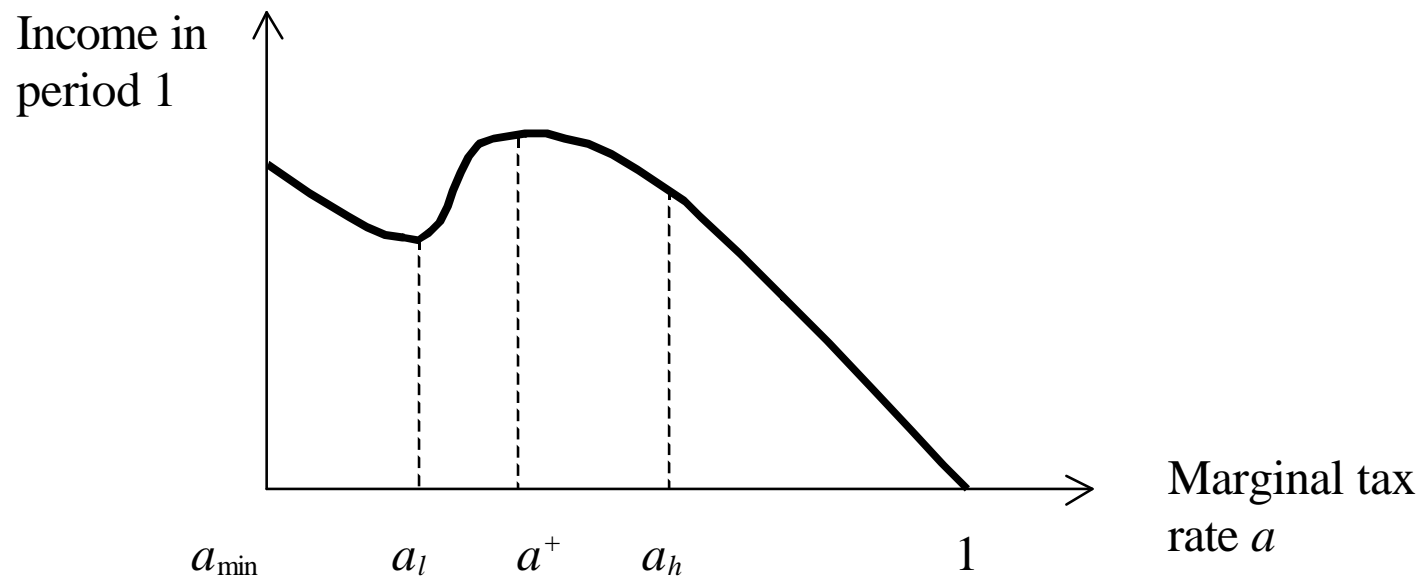

Figure 1. Relationship between $a$ and mean income in period 1. The arrow in the first panel indicates the direction in which mean income in period 1 increases. 